\title{
THE AMERICAN ORTHOPSYCHIATRIC ASSOCIATION, INC. I945 MEMBERSHIP LIST
}

The symbol following the address indicates membership status: (C) Charter member; (F) Fellow; (H) Honorary. Numbers in parenthesis indicate year of election to membership. The chief organization connection is followed by the mailing address, which may be either the home address or that of the organization.

* The asterisk indicates military service.

Absate, Grace McL., M.D., Bur. Child Guidance, I4I Joralemon St., Brooklyn 2, N. Y. (F) ('4I) Аввот, E. Stanley, M.D.; P.O. Box I 19 , Wayland, Mass. (F) ('29)

Acker Ly, S. Spafford, M.D.; Mental Hyg. Clinic, 6ro S. Floyd St., Louisville 2, Ky. (F) ('3I)

Ackerman, Nathan W., M.D., Jewish Bd. Guardians; 43 E. 78 th St., New York 21 (F). ('39)

Adams, Walter A., M.D., Provident Hosp.; 6216 Evans Ave., Chicago 37, Ill. (F) ('35)

Adamson, Elizabeth I., M.D.; Children's Court, $4_{24}$ County Office Bldg., White Plains, N. Y. (F) ('42)

Alexander, Franz, M.D.; Inst. for Psychoanalysis, 43 E. Ohio St., Chicago I1, Ill. (F) ('3I)

Alren, Cornelia H., Univ. of Buffalo, Sch. Soc. Work; I95 Voorhees Ave., Buffalo I4, N. Y. (F) ('3o)

Allen, Frederick H., M.D.; Child Guid. Clinic, i7I I Fitzwater St., Phila. 46, Pa. (F) ('26)

Alpern, Evelyn, M.D.; 545 Breckenridge St., Buffalo, N. Y. (F) ('33)

Alpert, Augusta, Ph.D., Mt. Sinai Hospital; 105 E. 38 th St., New York I6 ('40)

Amster, Mrs. Fanny; 59 Io Fordham, Houston 5, Texas (F) ('39)

*Anderson, Forrest N., M.D.; Major, MC, AUS, O'Reilly General Hosp., Springfield, Mo. (F) ('29)

Anderson, Harold H., Ph.D.; Dept. Psychology, University of Illinois, Urbana, Ill. (F) ('39)

Anderson, Rose G., Ph.D.; Psychological Service Center, 522 Fifth Ave., New York 18 (F) ('37)

Anderson, V. V., M.D; The Anderson School, Staatsburg-on-Hudson, N. Y. (F) (C)

Andrus, Ruth, Ph.D.; State Dept. of Education, Albany, N. Y. ('45)

Aptexar, Herbert H., Foster Home Bur.; 67-125 Dartmouth St., Forest Hills, N. Y. (F) ('39)

Arkin, Frances S., M.D.; 572 Park Ave., New York ('39)

Armstrong, Elizabeth W., Children's Center; 723 Seyburn Ave., Detroit, Mich. ('40)

Arrington, Winifred W., N.Y.C. Comm. on Mental Hyg.; 621 W. 17Ist St., New York 32 (F) ('30)

Arthur, Grace, Ph.D.; IO44 Lowry Medical Arts Bldg., St. Paul 2, Minn. (F) ('32)

*Aren, Eugene L., M.D.; Lt. Comdr., MC, USNR; c/o Child Guid. Clinic, 3605 Cedar Springs, Dallas 4, Texas (F) ('38)

*August, Harry E., M.D.; Major, MC, AUS, Consult. Serv., IRTC, Camp Blanding, Fla. (F) ('33)

Barbee, Margaret; Child's Foster Home Serv., 305 E. 86th St., New York 28 ('33)

Barger, William C., M.D.; Bur. Child Guidance; 30 Old Field Lane, Great Neck, L. I., N. Y. ('42)

*Barhash, A. Z., M.D.; Major, MC, AUS, Mason General Hosp., Brentwood, L. I., N. Y. ('42)

Bartelme, Phyllis, Ph.D., U. S. Employment Serv.; 5 Panoramic Way, Berkeley 4, Calif. (F) ("2g)

Bartemeier, Leo H., M.D., Henry Ford Hosp., 57 Cloverly Rd., Grosse Pointe Farms 30, Mich. (F) ('29)

Baruch, Dorothy W., Ph.D.; 3250 Country Club Dr., Los Angeles 6, Calif. (F) ('40)

Baschnagel, Catherine; 825 Park Ave., Plainfield, N. J. ('43)

Bassett, Clara; John Sealey Clinic, gth St. and Ave. B, Galveston, Tex. (F) ('28)

Beaghler, H. Edward, M.D.; I 107 N. Naomi, Burbank, Calif. ('43)

BEck, Samuel J., Ph.D.; Michael Reese Hosp., 29th St. \& Ellis Ave., Chicago I6, Ill. (F) ('29)

Beckmann, J. William, M.D., Bur. Child Guidance; 45 Prospect Place, New York 17 (F) ('4I)

BEELEY, Arthur L., Ph.D.; University of Utah, Salt Lake City 2, Utah (F) ('37)

Belcher, Esther L., Dept. Pub. Instruction, Special Ed. Div.; 535 Glenmore, East Lansing, Mich. ('40)

Bender, Lauretta, M.D.; Psychiatric Div., Bellevue Hosp., First Ave. and 3oth St., New York (F) ('37) 
*Benton, Arthur L., Ph.D.; Lt. Comdr., USNR, Naval Training Sta., Newport, R. I. (F) ('38)

Beres, David, M.D., Foster Home Bur.; 59 E. 79 th St., New York 21 ("44)

Berlin, Mrs. Caroline J.; 57 I I Pocusset St., Pittsburgh I7, Pa. ('32)

*Berman, Sidney, M.D.; Capt., MC, AUS, AAF Convalescent Hosp., Fort Logan, Colo. ('43)

Bernard, Viola W., M.D., Bur. Child Guidance; 930 Fifth Ave., New York 21 ('42)

Berner, Frank, M.D., College of P. \& S., Columbia Univ.; 133 E. 58 th St., New York 22 ('41)

*Binder, Harold J., M.D.; Capt., MC, AUS, Station Hospital, Ft. Oglethorpe, Ga. ('42)

Blanchard, Myron B.; National Jewish Welfare Bd., 145 E. 32nd St., New York I6 (F) ('34)

Blanchard, Phyllis, Ph.D.; Child Guid. Clinic, i7 I I Fitzwater St., Phila. 46, Pa. (F) ('30)

*Blau, Abram, M.D.; Lt. Comdr., MC, USNR, Naval Rec. Barracks, Lido Beach, L. I., N. Y. (F) ('39)

Buos, Peter, Ph.D., Personnel Bur., Brooklyn College; 820 Park Ave., New York 2I ('44)

Bone, Harry, Ph.D.; 400 W. I Igth St., New York 27 ('43)

Bradley, Charles, M.D.; Emma Pendleton Bradley Home, Riverside I 5, R. I. (F) ('36)

Branham, V. C., M.D.; Woodbourne Inst. for Defective Delinquents, Woodbourne, N. Y. ('42)

Brenman, Margaret, Ph.D.; The Menninger Clinic, Topeka, Kan. ('44)

Brickner, Ruth P., M.D., Child Study Assoc. of America; 4926 Goodridge Ave., New York 63 ('34)

Bridgman, Olga, M.D., Dept. of Pub. Health; I380 Monterey Blvd., San Francisco 16, Calif. (F) ('25)

BridGMAN, Ralph P.; Hampton Inst., Hampton, Va. (F) ('3I)

Brinker, Dorothy L., Martha H. Beeman Child Guid. Clinic; Jefferson \& 3rd St., Niagara Falls, N.Y. (F) ('32)

Broadwin, Isra T., M.D., C.C.N.Y., School of Education; 116 W. 59th St., New York 19 (F) ('30)

Brockbank, Thomas W., M.D.; Catholic Charities Guid. Inst., I33 E. 58th St., New York 22 (F) ('33)

*Bromberg, Norbert, M.D.; Lt., MC, USNR, Naval Repair Base Dispensary, New Orleans, La. ('43)

*Bromberg, Walter M., M.D.; Lt. Comdr., MC, USNR U. S. Naval Prison, Navy Yard, Portsmouth, N.H. ('41)

Bronner, Augusta F., Ph.D.; Judge Baker Guid. Center, 38 Beacon St., Boston 8, Mass. (F) ('25)

*Brown, Frederick W., Lt., AUS; 36 Wellington Rd., Garden City, N. Y. ('28)

Brown, Muriel W., Ph.D.; U. S. Office of Education, Washington 25, D. C. (F) ('30)

Bumstead, Kate S.; Pleasantville, Pa. ('44)

Burling, Temple, M.D.; Child Guidance Clinic, soo N. Main St., Providence 3, R. I. (F) ('33)

Burton, Aileen C.; Hotel Irving, New York 3 ('42)

Buxbaum, Edith, Ph.D.; 210 E. 68th St., New York 21 ('43)

Buxton, Rex E., M.D., Washington Inst. for Mental Hyg.; 5315 Worthington Dr. N.W., Washington 16 , D. C. (F) ('40)

Cameron, Eugenia S., M.D., State Bd. Health; 3507 Sunset Dr., Shorewood Hills, Madison 5, Wis. (F) ('29)

Camprell, Douglas G., M.D.; 160 Palo Alto Ave., San Francisco 14, Calif. ('40)

Carroll, Clara; Bur. Child Guidance, Queens Unit, 166th St., \& 65th Ave., Flushing, N. Y. ('42)

Carter, Jerry W., Jr., Ph.D.; Child Guid. Center, 3422 E. Douglas, Wichita 8, Kan. ('4I)

CARY, Mrs. Richard; R.D. River Road, Lewiston, N. Y. (F) ('3I)

*Cassino, Frank A., M.D.; Capt., MC, AUS, Walter Reed General Hosp., Washington, D. C. ('43)

*Challman, S. Alan, M.D.; Lt. Col., MC, Chief Surgeons Office, Hq. USASOS, APO 50I, c/o Postmaster, San Francisco, Calif. (F) ('35)

Chamberlain, Herbert E., M.D.; P.O. Box 933, Sacramento, Calif. (F) ('27)

Chapman, Ross McC., M.D.; Sheppard \& Enoch Pratt Hosp., Towson 4, Md. (F) ('28)

Chassell, Joseph, M.D.; Bennington College, Bennington, Vt. (F) ('38)

Chennell, Eileen A., M.D.; Military Hosp. (Women) Northampton, England ('44)

ChILders, Asher T., M.D.; Central Ment. Hyg. Clinic; 721 Berkeley Ave., Cincinnati 29, Ohio (F) ('3I)

*Chornyak, John, M.D.; Major, MC, Station Hosp., Truax Field, Madison 7, Wis. (F) ('38)

CHOv, Li-Chiu; Dept. of Sociology, Ginling College, Chingtu, West China ('36)

CLARK, Josephine E.; Guidance Center, 1737 Prytania St., New Orleans 13, La. ('42)

Clark, Mary Augusta; Bedford, N. Y. (F) ('30) 
Clemence, Esther H., Inst. for Juvenile Research; 1037 Cherry St., Winnetka, Ill. ('45)

Cirrton, Eleanor, Community Service Soc.; 15 W. I I th St., New York II ('27)

Clothier, Florence, M.D.; New England Home for Little Wanderers, 16I S. Huntington Ave., Boston 30, Mass. (F) ('42)

Cochran, Mrs. Mary Lue; Topaz Relocation Center, Topaz, Utah ('31)

Cconill, Havrie, deJ., M.D.; Chn's Mem. Clinic, I00I E. Clay St., Richmond I9, Va. (F) ('28)

Cohen, Anna, N. Y. Assoc. for Jewish Children; 80 Aycrigg Ave., Passaic, N. J. ('4I)

*Coleman, Jules V., M.D.; Major, MC (0-486259) 38th Div., APO 38, c/o Postmaster, San Francisco, Calif. ('39)

Conn, Jacob H., M.D., Johns Hopkins Hosp.; 2325 Eutaw Place, Baltimore 17, Md. (F) ('40)

"Coox, Stuart W., Ph.D.; Capt., AC, Psychological Res. Project, Langley Fieid, Va. ('43)

Cooper, Olive A., M.D., Child Guidance Clinic; I6o Maple St., Springfield 5, Mass. ('41),

Corman, Bertha, Jewish Children's Bur.; 5519 Drexel Ave., Chicago 37, Ill. ('42)

*Corson, Harold F., M.D., Major, MC, AUS; c/o Box 145, Stockbridge, Mass. ('31)

Cotrington, Frances, M.D.; 40 E. 61st St., New York 21, N. Y. ('4I)

Coucr, Mildren Warden, M.D.; Cromwell Hall, Cromwell, Conn. (' 31 )

Crampton, Jessie E.; Child Guid. Centre, 201 Montague St., Brooklyn 2, N. Y. (F) ('4I)

Crane, Harry W., Ph.D.; Drawer 809, Chapel Hill, N. C. ('37)

CRothers, Bronson, M.D.; Childrens Hosp., 300 Longwood Ave., Boston 15, Mass. (F) ('4I)

Crowedl, Sarah E.; Rudaho Ranch, Los Cruces, New Mexico ('36)

Crutcher, Hester B.; Dept. Mental Hygiene, State Office Bldg., Albany i, N. Y. (F) ('4I)

Crutcher, Roberta, M.D., Pasadena Child Guid. Clinic; 3875 Wilshire Blvd., Los Angeles 5, Calif. ('45)

Cunningham, J. M., M.D.; Bur. of Mental Hygiene, State Dept. Health, Hartford 6, Conn. (F) ('33)

Curtis, William B., M.D.; Psychiatric Serv. in Community, 64 Trumbull St., New Haven ro, Conn. (F) ('4I)

*Davidorf, Eugene, M.D., Major, MC, Ft. Leonard Wood, Mo.; c/o 321 So. Madison Ave., Lebanon, Mo. ('42)

Davis, Anne E., Inst. for Juvenile Research; 5532 Kenwood Ave., Chicago 37, Ill. (F) ('4I)

Davis, Margaret S., Welfare Council; 2 Glenview Ave., Toronto, Canada (F) ('34)

Dawlex, Almena; Child Guid. Clinic, i7 I Fitzwater St., Phila. 46, Pa. (F) ('31)

DEBERrY, Ellett M., M.D.; Sonora, Tex. (F) ('29)

Despres, Marian A., Ph.D.; 1220 E. 56th St., Chicago 37, Ill. ('39)

Deutsch, Regina, Bur. Child Guidance; 2010 Grand Ave., New York 43 ('43)

Dicks, Grace H.; Am. Red Cross, Military \& Naval Welf. Serv., 230 Spring St., N.W., Atlanta 3, Ga. (F) ('37)

Dimmick, Graham B., PhD.; Child Guidance Serv., 217 N. Upper St., Lexington I 5, Ky. ('43)

Dolan, Dorothea L., Mental Hygiene Clinic, 1035 E. Morehead, Charlotte 3, N. C. ('42)

Dole, Edgar A., Ph.D.; The Training School, Vineland, N. J. (F) ('25)

Dombey, Edith H.; Juvenile Court, E. 22nd St. \& Cedar Ave., Cleveland I 5, O. ('34)

Dominou Ez, Kathryn E.; Children's Service Center, 335 S. Franklin St., Wilkes-Barre, Pa. ('45)

Doniger, Simon, Ph.D.; Jewish Child Guidance Bur., 682 High St., Newark 2, N. J. ('41)

Doody, William M., M.D.; Catholic Charities of N. Y., Ig Bentley Ave., Jersey City 4, N. J. ('35)

Dummer, Mrs. W. F.; 679 N. Michigan Ave., Chicago 11 , Ill. (H) ('26)

Dunbar, Flanders, M.D.; Presbyterian Hosp., 3 E. 6gth St., New York 21 ('43)

DurfeE, Marion B., M.D.; Child Guidance Clinic, 21 Catherine St., Worcester 5, Mass. ('43)

Durkin, Helen E., Ph.D., New Rochelle Child Guid. Clinic; 7 Fairview Rd., Scarsdale, N. Y. ('42)

Dybwad, Gunnar; State Dept. Social Welf., 230 N. Grand Ave., Lansing 4, Mich. ('42)

*Ebaugh, Franklin G., M.D.; Col. MC, 8th Serv. Command, Santa Fe Bldg., Dallas 2, Tex. (F) ('29)

EIsler, Edwin R., M.D.; Inst. for Psychoanalysis, 43 E. Ohio St., Chicago II, Ill. (F) ('29)

"Elkınd, Henry B., M.D.; Major, MC, AUS (Address Unavailable) (F) ('28)

Emer y, E. Van Norman, M.D.; 4668 Pershing Ave., St. Louis 8, Mo. (F) ('3I)

Englander, Charles, M.D.; 4 I Hillside Ave., Newark 8, N. J. (F) ('3I)

Ephron, Harmon S., M.D.; 295 Central Park W., New York 24 (F) ('38)

Epstein, Morris D., M.D.; 49 E. 96th St., New York 28 ('4I) 
ERIKson, Erik H.; Inst. Child Welf,, Univ. of Calif,; 25 Hacienda Rd., Orinda, Calif. (F) ('42) Estes, Stanley G., Ph.D., Northeastern University; 60 Pinckney St., Boston 14, Mass. (" 4 I) Evans, Chester E.; Personnel Dept., Gen. Motors Corp., 5140 Riopelle, Detroit II, Mich. ('45) Ewerhardt, Paul J., M.D. Children's Hosp.; IO28 Connecticut Ave., N.W., Washington, D. C. (F) ('30)

Fabian, Abraham A., M.D., Brooklyn Child Guid. Centre; 15 E. Ioth St., New York 3 ('45)

*Falstein, Eugene I., M.D.; Lt. MC, USNR, U. S. Naval Hosp., Norman, Okla. ('39)

Feder, Leah, Ph.D.; Family Society, 400 Community Fund Bldg., Pittsburgh, Pa. (F) ('39)

Fendason, Mrs. L. S.; Univ. of Minnesota, 8 Jones Hall, Minneapolis I4, Minn. (F) ('37)

Fenton, Norman, Ph.D.; Box K-88, Kent Field, Calif. ('37)

Ferguson, W. G., M.D.; Martha H. Beeman Clinic, 650 Memorial Parkway, Niagara Falls, N. Y. (F) ('30)

Finley, Malcolm H., M.D., Girls H. S.; 290 Brentwood Ave., San Francisco 16, Calif. (F) ('37)

FIror, Marion, M.D.; Child Guid. Clinic, I 325 W. Adams Blvd., Los Angeles 7, Calif. ('42)

Fishback, Dora, M.D.; 43 E. Ohio St., Chicago II, Ill. ('44)

Fitzher bert, Florence, Community Serv. Society; 130 E. 67th St., New York ('43)

Firz-Simmons, Marian J., Ph.D., Grosse Pointe Board of Ed.; 1 1 30 Parker Ave., Detroit 14, Mich. (F) ('37)

*Fleming, Alice M. R.; Ens. W.V(S)(H) USNR, Naval Hosp., Newport, R. I. ('42)

Font, Marion McKenzie; Tulane Univ. Med. School, I430 Tulane Ave., New Orleans, La. ('4I)

Foster, Roberta; Amherst H. Wilder Child Guid. Clinic, 279 Rice St., St. Paul 2, Minn. ('4I)

Foster, Sybil; I-A Chateau Lafayette, Greenwich, Conn. (F) ('30)

Foxe, Arthur N., M.D.; 25 West 54 th St., New York 19 ('41)

Francis, Mrs. Blythe W.; Family Welf. Assoc., 126 W. 3rd St., Los Angeles I3, Calif. (F) ('37)

Frank, Lawrence K.; 72 Perry St., New York 14 (F) ('32)

FrankL, Mrs. Anni W.; 5 I 5 Richmond Ave., Buffalo I3, N. Y. ("42)

FrankL, George, M.D.; Guidance Center, 88 Goodell St., Buffalo 3, N. Y. ('44)

Frankley, Greta, M.D.; 350 Central Park W., New York 25 ('39)

French, Lois Meredith; 98 Alexander Ave., Nutley, N. J. (F) ('33)

*Friend, Maurice R., M.D., Lt., M.C.; c/o Dr. Emanuel London, IgoI Ave. N, Brooklyn 30, N. Y. ('43)

Fries, Margaret E., M.D.; 2I W. 86th St., New York 24 (F) ('36)

FRY, Clements C., M.D.; Iog College St., New Haven, Conn. (F) ('3I)

Fuller, Earl W., M.D., Northern N. J. Ment. Hyg. Clinics; 8 Mill Rd., Morris Plains, N. J. (F) ('3I)

Gabell, Marcene P.; Box 216, South Yarmouth, Mass. ("42)

*Gaw, Emir A., M.D.; Lt., MC, USNR, c/o 4319 Trias St., San Diego 3, Calif. ('43)

*Gardner, George E., M.D., Lt. Comdr. MC, USNR; c/o 7 Trapelo Rd., Belmont, Mass. (F) ('4I)

Garretr, Annette; Smith College Sch. for Soc. Work, 8 College Hall, Northampton, Mass. (F) (' 3 I)

Gartland, Ruth, Sch. Applied Soc. Sci., Univ. of Pittsburgh; I033 Devonshire Rd., Pittsburgh I3, $\mathrm{Pa}$. ('42)

Geleerd, Elisabeth R., M.D.; 44 E. 8oth St., New York ('43)

Gerard, Margaret W., M.D.; 5744 Harper Ave., Chicago 37, Ill. (F) ('3I)

Gesell, Arnold, M.D.; Clinic of Child Devel., I4 Davenport Ave., New Haven I I, Conn. (F) ('3I)

Gilbert, Louis Jay, M.D., Bur. Child Guidance; 235 E. 22nd St., New York ('44)

GildeA, Margaret C.-L., M.D., Washington Univ. Sch. of Med.; 4952 Maryland Ave., St. Louis 8, Mo. ('44)

Ginssurg, Mrs. Ethel L., N.Y.C. Comm. on Mental Hyg.; 2 E. 97th St., New York 29, (F) ('39) Ginsburg, Sol W., M.D., Mt. Sinai Hosp.; 12 E. 97th St., New York 29 ('45)

Gitelson, Maxwell, M.D.; Michael Reese Hosp., 29th St. \& Ellis Ave., Chicago I6, Ill. (F) ('36)

Glatzer, Henriette T., Brooklyn Child Guid. Centre; 30 Disbrow Lane, New Rochelle, N. Y. ('42)

Glauber, Helen M.; 829 Park Ave., New York 21 ('4I)

Glickman, Martha H., Ph.D.; I34 Haven Ave., New York 34 ('33)

*Goldensohn, Leon N., M.D.; Major, MC (0-503397) APO 4 IO, c/o Postmaster, New York ('42)

Goldberg, Milton, M.D., Inst. for Juv. Research; 1028 E. Hyde Park Blvd., Chicago I 5, Ill. ('45)

*Goldfarb, William, Ph.D., USPHS(R); 720 Riverside Dr., New York ('45) 
Goldman, Julia, N. Y. Assoc. for Jewish Children; 1230 Park Ave., New York (F) ('33)

*Goldman, Nathan; Lt. Comdr, MC-H (S) USNR, U. S. Naval Disciplinary Barracks, Navy Yard, Phila. 12, Pa. ('39)

*Goodman, Soll, M.D.; Lt. Col., MC, Consultation Serv., Hq. ASFTC, Camp Lee, Va. ('44)

Gould, Miriam C.; Vassar College, Poughkeepsie, N. Y. ('33)

Green, Rose; Univ. of Minnesota, Dept. Sociology \& Soc. Work, Minneapolis I4, Minn. (F) ('38)

Greenacre, Phyllis, M.D., New York Hosp.; 970 Park Ave., New York 28 ('43)

Grifrith, Mrs. Carleton, State Dept. Pub. Welf,; ro5o Logan St., Denver 3, Colo. ('42)

*Grossman, Royal G., M.D., Col., MC; c/o 1423 Arthur Ave., Lakewood, O. ('28)

Grossmann, Grace; Nat'l Council Jewish Women, 285 Schermerhorn St., Brooklyn 17, N. Y. (F) ('3I)

Groves, Ernest R., University of North Carolina, Box 929, Chapel Hill, N. C. (F) ('4I)

HACker, Frederick J., M.D.; 247 S. Beverly Dr., Beverly Hills, Calif. ('45)

Hagan, Margaret W., Am. Red Cross, St. Elizabeth's Hosp.; 305 Tenth St., N.E., Washington, D. C. (F) ('36)

*Hagman, Elmer R., Ph.D., Lt. USNR; 3475 Ullman St., San Diego 6, Calif. (F) ('33)

Halpern, Florence, Bellevue Hosp., Psychiatric Div.; 222 E. 82nd St., New York 28 ('44)

Hamilton, E. Louise, Ph.D.; 1828 W. Tioga St., Phila. 40, Pa. ('39)

Hankins, Dorothy; Child Guidance Clinic, I7I I Fitzwater St., Phila. 46, Pa. (F) ('40)

Hannetr, Frances, M.D., Univ. of Chicage Health Serv.; 5434 Hyde Park Blvd., Chicago I 5 , Ill. (F) ('39)

Hanson, Harold B., M.D., U. S. Children's Bur.; 1995 Bayard Ave., St. Paul 5, Minn. (F) ('39)

Hardcastle, Dorothy H., Mental Health Emergency Comm.; 2 Lyndhurst Rd., Hampstead, London N.W. 3, England ('4I)

Hardcastle, Douglas N., M.D., Ministry of Health; 48 Warwick Rd., Bishops Stortford, Hertfordshire, England ('34)

Harle, Marjorie; Guidance Center, 3 I Gibbs St., Rochester 4, N. Y. ('44)

*Harms, Herbert E., M.D.; Major, MC, Mental Hygiene Unit, MacDill Field, Tampa 8, Fla. ('4I)

Harris, June; Bureau of Child Guidance, 228 E. 57th St., New York 22 ('42)

HARrison, Frances; Norristown State Hospital, Norristown, Pa. ('32)

Hartwell, S. W., M.D., Univ. Buffalo Med. Sch.; I76 Washington Highway, Snyder, N. Y. (F) ('29)

Healy, William, M.D; Judge Baker Guidance Center, 38 Beacon St., Boston 8, Mass. (F) (C)

Heath, Esther, Pasadena Child Guidance Clinic; 107 Grace Terrace, Pasadena 2, Calif. ('45)

HeLdT, Thomas J., M.D.; Henry Ford Hosp., Detroit 2, Mich. ('29)

*Helgesson, Uno H., M.D.; Comdr. USNR, U. S. Navy Yard, Boston, Mass. ('3I)

Hennessy, Maurice A. R., M.D.; 1051 5 Carnegie Ave., Cleveland 6, O. ('31)

Henry, Jules, Ph.D., Office of Inter-American Affairs; 3640 Gunston Rd., Alexandria, Va. ('43)

Hers kowitz, Herbert, M.D.; Norristown State Hosp., Norristown, Pa. ('44)

Hertz, Marguerite R., Ph.D., Western Reserve Univ.; 2835 Drummond Rd., Cleveland 20, 0. ('42)

Hertzman, Jack M.D., Board of Education; 19 Garfield Place, Cincinnati 2, O. ('39)

Hicks, Helen R.; 5400 Whittaker Road, Ypsilanti, Mich. ('4I)

HrLL, Julia Ford, M.D.; Child Guidance Center, 3604 Victoria St., Pittsburgh 13, Pa. (F) ('35)

HiLl, Lewis B., M.D., Smith College Sch. for Soc. Work; Io W. Madison St., Baltimore I, Md. (F) ('38)

Hincks, C. M., M.D.; Canadian Nat'l Comm. for Ment. Hyg., in I St. George St., Toronto, Can. (F) ('33)

Hincks, Elizabeth M., Ph.D.; 5 I Brattle St., Cambridge 38, Mass. ('32)

Hirsch, Jeannette S.; 24I Central Park W., New York 24 ('42)

Hochreiter, Mrs. F. C.; 2 II 8 Treasure St., New Orleans 19, La. ('4I)

Holmer, Paul M.D., Children's Hosp. of East Bay; 1079 Ashmount Ave., Oakland 1o, Calif. (F) ('34)

*Holt, William L., Jr., M.D.; Lt. Comdr, MC, USNR; c/o 35 William St., Geneva, N. Y. ('40)

*Holzberg, Jules D., Lt. AUS, Mason Gen. Hosp., Brentwood, N. Y.; c/o I 128 Findlay Ave., Bronx, N. Y. ('45)

*Horton, Charles B., M.D.; Major, MC, Gulf Stream Hosp., Miami Beach, Fla. (F) ("33) 
Houtz, Mrs. Philip, Jewish Board of Guardians; 124 W. 79th St., New York ('44)

Humphreys, Edward J., M.D.; Bur. Ment. Hyg., Div. Ment. Dis., State Office Bldg., Columbus 16, O. ('45)

Irons, Lucia, Am. Red Cross; c/o Calvert Court, Apt. D8, Baltimore I8, Md. ('39)

Itrleson, Mrs. Blanche; 965 Fifth Ave., New York 21 (H) ('40)

JAckson, Edith B., M.D.; Dept. Pediatrics, Yale Univ. Med. Sch., 789 Howard Ave., New Haven 4, Conn. (F) ('38)

Jackson, James D.; 27 A North Dean Ave., Trenton 8, N. J. ('30)

JAEnIKE, R. C. A., M.D.; Child Guidance Clinic, Strong Memorial Hosp., Rochester 7, N. Y. ('33)

January, Mildred H., M.D., Board of Ed.; i i I Gillett St., Hartford 5, Conn. ('44)

Janvier, Carmelite, Div. Special Serv., Pub. Sch.; 3307 Prytania St., New Orleans I 5, La. (F) ('35)

Jefrerson, R. A., M.D., Marquette Univ. Sch. Med.; 208 E. Wisconsin Ave., Milwaukee 2, Wis. ('33)

Jenkins, R. L., M.D.; Inst. for Juv. Research, 907 S. Wolcott Ave., Chicago 12, Ill. (F) ('39)

Jessner, Lucie, M.D., Habit Clinic for Child Guidance; I07 Garden St., Cambridge 38, Mass. ('45)

Johnson, Adelaide M., M.D., Inst. for Psychoanalysis; 5807 Dorchester Ave., Chicago 37, Ill. (F) ('4I)

Johnson, Eleanor H., Ph.D.; 35r Farmington Ave., Hartford 5, Conn. (F) ('26)

Johnson, George S., M.D.; Stanford Univ. Hosp., Clay \& Webster Sts., San Francisco 15, Calif. (F) ('30)

Jones, Harold E., Ph.D., Inst. Child Welf., Univ. of Calif.; 2683 Shasta Rd., Berkeley 8, Calif. (F) ('38)

Joslyn, June J.; Oregon Mental Hygiene Society, Pittock Block, Portland, Ore. (F) ('34)

Karpman, Benjamin, M.D.; St. Elizabeth's Hospital, Washington, D. C. ('39)

Kasanin, Jacob, M.D.; Mt. Zion Hosp., Post \& Scott Sts., San Francisco I 5, Calif. (F) ('26)

Kaufman, S. Harvard, M.D.; Western State Psychiatric Hosp., DeSoto \& O'Hara Sts., Pittsburgh I 3, Pa. ("44)

Kawin, Ethel, Glencoe Public Schools; 1725 E. 53rd St., Chicago I5, Ill. (F) ('32)

KeIser, Sylvan, M.D., Bellevue Hospital; 3 E. 85 th St., New York 28 ('43)

*Kemble, Robert P., M.D.; Major, MC, 3384 th SU, S.T.U., New Cumberland, Pa. ('40)

Kenworthy, Marion E., M.D.; 1035 Fifth Ave., New York 28 (F) ('24)

Kimbald, Everett, Ph.D.; College Hall 8, Smith College, Northampton, Mass. (F) ('30)

King, Anna, Austen Riggs Foundation, Inc.; Yale Hill, Stockbridge, Mass. ('42)

Kirkpatrick, M. E., M.D.; Guidance Center, 1737 Prytania St., New Orleans I3, La. (F) ('3I)

KleIN, Emanuel, M.D., Bur. Child Guidance; 47 E. 88th St., New York (F) ('40)

KLeinschmidt, Sara; Bur. Crippled Children, Board of Health, Honolulu I, T. H. ('40)

*KNapp, Irving J., M.D., Capt. MC, AUS; c/o I La Veta Place, Nyack, N. Y. ('37)

Косн, Eleanor S., M.D.; I64o Pine St., Philadelphia, Pa. ('42)

Kovarsky, Marcel; Jewish Child Welf. Assoc., I 220 Chemical Bldg., St. Louis I, Mo. (F) ('3I)

KRIs, Ernst, Ph.D., New School for Social Research; 135 Central Park West, New York 23 ('44)

Krug-Brady, Othilda, M.D.; Central Ment. Hyg. Clinic, General Hosp., Cincinnati, O. ('44)

Krugman, Morris, Ph.D.; Bur. Child Guidance, 228 E. 57 th St., New York 22 (F) ('39)

Kundert, Elizabeth, M.D.; Mental Hygiene Clinics, 20 State St., Montpelier, Vt. ('44)

LaBine, Alfred C., M.D.; 8-259 General Motors Bldg., Detroit 2, Mich. ('28)

LaMar, Norvelle C., M.D., Payne Whitney Clinic, New York Hosp.; 139 E. 73 rd St., New York 2I (F) ('30)

*Lander, Joseph, M.D., Capt., MC; c/0 808 North Crescent Ave., Cincinnati 29, O. ('40)

Landis, Edward E., M.D.; Dept. Psychiatry, Gen. Hosp., 323 E. Chestnut St., Louisville 2, Ky. ('42)

Landis, Marjorie R., Allentown State Hosp.; 2929 Chew St., Allentown, Pa. ("42)

*Langford, William S., M.D.; Major, MC, AAF School of Aviation Med., Randolph Field, Tex. (F) ('36)

Langner, Helen P., M.D., New York Hosp.; 40 E. 6ist St., New York 2I (F) ('29)

Larson, John A., M.D.; 300I $\frac{1}{3}$ Grape St., San Diego, Calif. ('3I) 
Laughead, Mary B., Fordham Univ. School Soc. Serv.; 44 Gramercy Pk., No., New York 10 ('30)

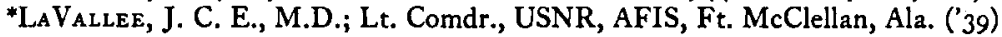

Lawton, George, Ph.D.; 4 I W. 82nd St., Apt. 4B, - New York 24 (F) ('35)

Layman, Mary H., M.D., Stanford Med. School; 21 56 Pacific Ave., San Francisco I5, Calif. ('42)

Ledwith, Mrs. W. M.; Child Guid. Center, 3604 Victoria St., Pittsburgh 13, Pa. ('45)

Leonard, Christina M., M.D., Fordham Univ. Sch. Soc. Serv.; 44 Gramercy Pk. No., New York 10 (F) ('24)

Leonard, Shirley; Bureau of Child Guidance, 228 E. 57th St., New York 22 (F) ('3I)

Levey, Harry B., M.D.; Inst. for Psychoanalysis, 43 E. Ohio St., Chicago I I, Ill. (F) ('38)

Levine, Maurice, M.D., Univ, of Cincinnati Med. Sch.; 984 Lenox Place, Cincinnati 29, O. (F) ('4I)

LevY, David M., M.D.; 136 E. $57^{\text {th }}$ St., New York 22 (F) (C)

Lewin, Bertram D., M.D.; 32 E. 64th St., New York 21 (F) ('31)

Lewis, Margaret, Assoc. Charities of Cleveland; Park Lane, Chagrin Falls, O. (F) (35)

*Lewis, Murray D., M.D.; Lt. MC, AUS, ASFTC, Ft. Lewis, Wash. ('42)

Lib bey, Betsey; Family Society, 3i i S. Juniper St., Phila. 7, Pa. ('3I)

Lion, Ernest G., M.D., Stanford Univ. Sch. of Med.; 490 Post St., San Francisco 2, Calif. ('40)

Lippman, H. S., M.D.; Amherst H. Wilder Child Guid. Clinic, 279 Rice St., St. Paul 2, Minn. (F) ('28)

Liss, Edward, M.D., New York School for Soc. Res.; 130 E. 39th St., New York 16 (F) ('32)

Lithauer, Donah B., N. Y. Assoc. for Jewish Children; 150 W. 95 th St., New York 24 ('32)

Litrle, Harry M., M.D.; Child Guid. Center, 3604 Victoria St., Pittsburgh 13, Pa. (F) ('35)

Lloyd, Ruth; 308 Commonwealth Ave., Boston, Mass. ('43)

Lloyd, Wilma; Inst. Child Welf., Univ. of California; 6roo Snake Rd., Oakland i , Calif. (F) ('3I)

Loewald, Hans W., M.D.; Mental Hyg. Soc. of Maryland, 6oI W. Lombard, Baltimore I, Md. ('43)

Long, Alroy S., Bureau of Child Guidance; 75 St. Nicholas Place, New York ('42)

Lorand, Sandor, M.D., Psychoanalytic Inst.; 40 Central Park So., New York 19 (F) ('33)

Lorr, George M., M.D., Suffolk County Dept. of Health; Box 66, Setauket, L. I., N. Y. ('33)

*Lourie, Reginald S., M.D.; Lt., USNR, U. S. Naval Convalescent Hosp., Glenwood Springs, Colo. ('4I)

Lowrey, Lawson G., M.D.; 25 W. 54 th St., New York 19 (F) (C)

LowrY, Fern; N. Y. School of Social Work, 122 E. 22nd St., New York 10 (F) ('37)

Lucas, Leon, Jewish Child Guid. Bur., Newark; 2210 Bronx Park E., Bronx 60, N. Y. ('44)

LuCKey, Bertha M., Ph.D.; Board of Ed., 6th \& Rockwell, Cleveland I 4 , Ohio (F) ('3I)

Luenrs, Leslie E., M.D.; 14 Washington Sq. N., New York i I ('29)

LurIE, Louis A., M.D., Child Guidance Home; I9 Garfield Place, Cincinnati 2, O. (F) ('26)

Lurie, Olga Rubinow, Ph.D., Jewish Soc. Serv. Bur.; 5606 S. Blackstone Ave., Chicago 37, 1ll. (F) ('39)

Lyman, Richard, M.D.; Duke University, Durham, N. C. (F) ('34)

McAlpin, Katharine B.; Child Guid. Clinic, 1325 W. Adams Blvd., Los Angeles 7, Calif. ('44)

McBee, Marian; N. Y. C. Comm. on Mental Hygiene, 105 E. 22nd St., New York 10 (F) ('39)

McClure, Mrs. R. H.; Child Guid. Center, 3604 Victoria St., Pittsburgh 13, Pa. (F) (' 31 )

McCord, Clinton P., M.D.; 54 Willett St., Albany 6, N. Y. (F) ('26)

McLean, Helen V., M.D.; Inst. for Psychoanalysis, 43 E. Ohio St., Chicago I I, Ill. (F) ('40)

McNIEL, Edwin E., M.D.; 3875 Wilshire Blvd., Los Angeles 5, Calif. ('37)

MacDonald, Martha W., M.D.; Bur. Mental Hygiene, Queens Hospital, Honolulu, T. H. (F) ('33)

MacDovgall, Elizabeth, M.D.; 1205 Scott Ave., Winnetka, Ill. ("44)

MacFarlane, Jean W., Ph.D.; 1220 Oxford St., Berkeley 7, Calif. (F) ('30)

MAEDer, LeRoy M. A., M.D., Psychoanaly tic Inst.; 206 S. I 3 th St., Phila. 7, Pa. (F) ('29)

Magiera, Estelle A., M.D.; Child Guid. Clinic, 704 Standard Life Bldg., Jackson 27, Miss. ("45)

Mahler, Margaret S., M.D.; 350 Central Park W., New York 25 ('45)

Malcove, Lillian, M.D., Psychoanalytic Inst.; 245 E. 72nd St., New York (' 31 )

Mann, Edna B., Bureau of Child Guidance; 215 W. 98 th St., New York 25 ('42)

*MarkeY, Oscar B., M.D.; L.t. Col. MC, APO 357, c/o Postmaster, San Francisco, Calif. (F) ('28)

Marguis, Dorothy P., Ph.D.; Yale Univ. School of Med., 333 Cedar St., New Haven I I, Conn. ('44)

Marshall, Berry C., M.D.; 419 Hawthorn Rd., Baltimore 10, Md. ('33) 
Marshall, Helen; University of Utah, Dept. of Psychology, Salt Lake City I, Utah ('34)

Martin, Alexander R., M.D.; 24 Gramercy Park, New York 3 (F) ('42)

Martin, A. Leila; Board of Ed., I 3 Fitzhugh St. S., Rochester 4, N. Y. (F) ('26)

Mathews, Julia; Child Guid. Clinic, I325 W. Adams Blvd., Los Angeles 7, Calif. ('44)

*Mathews, W. Mason, Ph.D.; Lt., USNR, U. S. Naval Hospital, Bethesda, Md. ('40)

Mayer, Edward E., M.D., Univ. of Pittsburgh; 3401 Fifth Ave., Pittsburgh, Pa. ('34)

MeAd, Margaret, Ph.D.; American Museum Natural History, I5 W. 77 th St., New York 24 ('45)

MekeEL, H. Scudder, Ph.D.; 328-B Sterling Hall, University of Wisconsin, Madison 6, Wis. ('45)

Mellor, Ruth; Mental Hygiene Clinic, 6ro S. Floyd St., Louisville 2, Ky. (F) ('39)

Meltzer, H., Ph.D., Psychological Serv. Center; 4510 Maryland Ave., St. Louis 8, Mo. (F) (' 31 )

Menaker, Esther, Ph.D.; 20 W. 77th St., New York 24 ('39)

Menger, Clara; Child Guidance Bur., soth \& Gaty, East St. Louis, Ill. ('43)

Menninger, Karl A., M.D.; The Menninger Clinic, Topeka, Kan. (F) (C)

*Menninger, William C., M.D.; Col., MC, Office of Surgeon Gen., 1818 H St. N.W., Washington 25, D. C. (F) ('35)

Meyer, Adolf, M.D.; 4305 Rugby Road, Baltimore 10, Md. (F) ('24)

*Michaels, Joseph J., M.D.; Capt., MC, Newton D. Baker Gen. Hosp., Martinsburg, W. Va. (F) ('38)

Miller, Joseph S. A., M.D.; Rockland State Hosp., Orangeburg, N. Y. ('4l)

Millet, John A. P., M.D., Presbyterian Hosp.; 770 Park Ave., New York 2I ("45)

Mirviss, Sophia, M.D., Mt. Zion Hosp.; 244 I Vallejo, San Francisco 23, Calif. ('44)

Mонr, Mrs. Esther J.; I 320 E. 56th St., Chicago 37, Ill. ('32)

Morr, George J., M.D.; Inst. for Psychoanalysis, 43 E. Ohio St., Chicago I , Ill. (F) ('26)

Монк, Irma E., Bur. of Attendance, Board of Ed.; 25 Minetta Lane, New York 12 (F) ('40)

Montague, Helen, M.D.; 27 W. gth St., New York (F) ('24)

Montcomery, Elizabeth T.; 1026 Pine St., Winnetka, Ill. ('4I)

Moore, Madeline U.; Community Service Soc., 37-34 82nd St., Jackson Heights, N. Y. (F) ('33)

Moore, T. V., M.D.; St. Anselm's Priory, $1_{4}$ th St. \& S. Dakota Ave. N.E., Washington 17, D. C. (F) ('4I)

Moore, William E., M.D.; Child Guidance Center, 312 Locust St., Akron, O. ('44)

Morsh, Mrs. Edith S.; 5570 Chancellor Blvd., Vancouver B. C., Canada ('28)

Moulton, Bryant E., M.D.; 18 Symmes Rd., Winchester, Mass. ('3I)

Mountain, Johanna C. G., Presbyterian Hosp.; 423 W. 1 2oth St., New York 27 ('42)

Moyle, Henry B., M.D.; Hartley-Salmon Clinic, 79 Farmington Ave., Hartford 5, Conn. (F) ('29)

Murphey, Bradford J., M.D., Univ. of Colo., College of Med.; 814 Republic Bldg., Denver 2, Colo. (F) ('29)

Murray, Willa L.; P. O. Box 404, Hilo, T. H. ('30)

Nelson, William, M.D.; 5449 Delmar Blvd., St. Louis, Mo. ('29)

Ness, Claire M., M.D., Bur. Ment. Hyg., State Dept. Health; 56 Garden St., Hartford 5, Conn. ('44)

Neumann, Frederika; Jewish Board of Guardians, 228 E. I gth St., New York 3 (F) ('3I)

*Neweld, H. Whitman, M.D., Lt. Col., MC; c/o 7005 Copeleigh Rd., Baltimore, Md. (F) (' 30 )

Nicholson, Marian B., Am. Red Cross, U. S. Naval Hosp.; 221 5 Marin Ave., Berkeley, Calif. ('40)

Nicolls, Mary M., San Diego City Schools; $35604^{\text {th }}$ Ave., San Diego 3, Calif. ('42)

Oberndorf, Clarence P., M.D.; 40 W. 59 th St., New York I9 (F) ('31)

O'BrieN, Frank J., M.D.; Board of Ed., I Io Livingston St., Brooklyn, N. Y. (F) ('25)

Olson, Elma, American Red Cross; 40 E. 72 nd St., New York 21 (F) ("29)

Orson, Willard C., Ph.D.; School of Ed., University of Michigan, Ann Arbor, Mich. (F) ('29)

O'Neill, Mrs. Claire H.; 9 10 Sir Francis Drake Blvd., San Anselmo, Calif. ('43)

Orgel, Samuel Z., M.D.; 667 Madison Ave., New York 21 (F) ('29)

${ }^{*} \mathrm{O}_{\mathrm{R} R}$, Douglass W., M.D.; Lt., MC, USNR, U. S. Naval Hospital, Astoria, Ore. ('42)

O'SheA, Harriet E., Ph.D.; Psychology Dept., Purdue University, Lafayette, Ind. (F) ('39)

PARKer, Z. Rita, M.D.; i Is E. 6Ist St., New York 2I ('28)

Patey, Henry C.; West Riding, Wellesley 81, Mass. ('40)

Patry, Frederick L., M.D.; 218 State St., Albany 6, N. Y. (F) ('32)

Paulsen, Alma A., Bur. Child Guidance; 416 W. 2oth St., New York in ('42)

Paynter, Richard H., Ph.D., Long Island University; 395 Grand Ave., Brooklyn I6, N. Y. (F) ('3o) 
Pearson, Gerald H. J., M.D., Temple Univ. Med. School; ir I N. 49th St., Phila., 39, Pa. (F) ('30)

Peppard, S. Harcourt, M.D.; Bur. Child Guidance, 228 E. 57th St., New York 22 (F) ('3I)

Perkins, Nellie L., Ph.D.; 208 Delaware Ave. W., Urbana, Ill. ('25)

Perry, Doris E.; 137 Scamman St., So. Portland, Me. ('30)

Polley, Jeannette M.; 4208 Sheridan Rd., Youngstown 8, O. ('32)

Porter, Betty, Family Welfare Assoc.; 706 Delaware St. S.E., Minneapolis 14, Minn. ('43)

Portser, Elinor G.; 423 E. Wister St., Germantown, Phila. 44, Pa. ('40)

Prather, Mrs. Helen G., American Red Cross; 828 Taylor St., San Francisco 2, Calif. ('43)

Pratt, George K., M.D., Conn. Society Ment. Hyg.; 80 Myrtle Ave., Westport, Conn. (F) ('27)

Preston, George H., M.D.; Bd. Mental Hygiene, 330 N. Charles St., Baltimore I, Md. (F) ('29)

Pritchetr, Henry L., Ph.D.; Box 503, Southern Methodist Univ., Dallas 5, Texas ('31)

Purnam, Marian C., M.D.; The Children's Center, 244 Townsend St., Roxbury 21, Mass. (F) ('32)

Quinlan, Gertrude; Catholic Charities Guid. Inst., I33 E. 58 th St., New York 22 (F) ('35)

Rabin, Albert I., Ph.D., State Hospital Clinics; 105 Pleasant St., Concord, N. H. ('44)

*Rademacher, E. S., M.D.; Lt. Comdr., U. S. Coast Guard Training Sta., Manhattan Beach, Brooklyn 29, N. Y. (F) ('27)

Rado, Sandor, M.D.; 25 E. 86th St., New York 28 (F) ('42)

*Ranck, Katherine H.; Lt., USNR, Navy Relief Society, Naval Air Station; Alameda, Calif. ('39)

Rank, Beata; Judge Baker Guidance Center, 38 Beacon St., Boston 8, Mass. ('4I)

Rapaport, David, Ph.D.; The Menninger Clinic, Topeka, Kan. ('44)

Raphael, Theophile, M.D., University of Michigan; I 520 Hill St., Ann Arbor, Mich. (F) ('26)

Rapoport, Jack, M.D., Bellevue Psychiatric Hosp.; I255 E. 2ist St., Brooklyn 30, N. Y. ('43)

REDL, Fritz, Ph.D., Wayne University; 17673 Manderson Dr., Detroit 3, Mich. (F) ('42)

Revve, George H., M.D., Western Reserve Univ.; 10465 Carnegie Ave., Cleveland 6, Ohio (F) ('26)

Reichard, Suzanne K., Homewood Terrace, San Francisco; I021 Keeler Ave., Berkeley 8, Calif. ('44)

Reichen berg, Wally, Ph.D.; Riverdale Country School, Riverdale-on-Hudson, New York 63 (F) ('38)

Reiman, M. Gertrude, Ph.D.; Child Center, Catholic Univ., Washington 17, D. C. ('42)

Reiss, Oscar, M.D., Univ. So. California, Sch. of Med.; 2200 W. 3rd St., Los Angeles, Calif. ('44)

Rernolds, Bertha C.; 3947-48th St., Long Island City 4, N. Y. (F) ('30)

*Reynolds, Chester L., M.D.; L.t. Comdr., USNR, U. S. Naval Hosp., Mare Island, Vellejo, Calif. ('40)

Rheingold, Harriet L., Inst. for Juvenile Research; 1102 E. 46th St., Chicago I 5, Ill. ('45)

Rich, Gilbert J., M.D.; Guidance Clinic, 515 Public Safety Bldg., Milwaukee 3, Wis. (F) ('29)

*Richards, T. W., Ph.D.; Lt. Comdr. USNR, U. S. Naval Hosp., San Leandro, Calif. ('4I)

*Richie, Richard F., M.D.; Major, MC.; c/o Boalsburg, Pa. ('39)

Richmond, Winifred V., Ph.D.; 20I E. Coronado Road, Santa Fe, N. Mex. (F) ('36)

Richter, Helen G., M.D.; P.O. Box 43, Narbeth, Pa. ('43)

Ridenour, Nina, Ph.D.; N.Y.C. Comm. on Mental Hygiene, Ios E. 22nd St., New York 10 (F) ('4I)

Rivuin, Harry N., Ph.D.; Queens College, Flushing, N. Y. (F) ('39)

RoвIE, Theodore R., M.D.; I 44 Harrison St., East Orange, N. J. ('30)

Robinson, Bruce B., M.D.; Bur. Child Guidance, 31 Green St., Newark 2, N. J. (F) ('29)

Robinson, J. Franklin, M.D.; Children's Serv. Center, 335 S. Franklin St., Wilkes Barre, Pa. ('42)

*Robinson, Lindsay E., M.D.; Major, MC; c/o 230 Lake St., Rouses Point, N. Y. ('37)

Rockwell, Alice J., Ph.D.; Ment. Hyg. Soc. of Maryland, 6oI W. Lombard St., Baltimore I, Md. (F) ('33)

Rogers, Carl R., Ph.D., 5844 Stoney Island Ave., Chicago 37, Ill. (F) ('34)

Roland, Mary C.; Tri-County Child Guid. Center, Ioog N. 2nd St., Harrisburg, Pa. ("42)

Romano, John, M.D.; Central Ment. Hyg. Clinic, Cincinnati General Hosp., Cincinnati, O. (44)

Root, June A.; 2543 Woodburn Ave., Cincinnati 6, O. (F) ('3I)

*Rose, John A., M.D.; Lt., MC, USNR., U. S. Naval Hosp., Oakland, Calif. ('4I)

Rosebrook, Wilda M., Ph.D.; Ohio State University, 321 Arps Hall, Columbus 10, O. ('40)

*Rosenheim, Frederick, M.D.; Capt., MC, 824 Engr. Bn. AVN (SEP), APO 610, c/o Postmaster, New York (F) ('39)

Rosenthal, Pauline, M.D., Bellevue Hospital, Psychiatric Div.; i 3 E. Igth St., New York ('40)

Rosenthal, Mrs. William H.; Child Guid. Home, 3149 Harvey Ave., Cincinnati 29, O. ('40) 
Rosenzweic, Leonard, M.D.; Warren State Hosp., Box 240, Warren, Pa. ('39!

Rosenzweic, Saul, Ph.D.; Western State Psychiatric Hosp., Pittsburgh I3, Pa. (F) ('35)

Ross, Helen, Inst. for Psychoanalysis; i 5 I E. 56th St., Chicago 37, Ill. (F) ('39)

Ross, Mabel, M.D.; Guidance Center, 88 Goodell St., Buffalo 3, N. Y. ('42)

Ross, Mrs. Mildred M.; 2329 S. Taylor Rd., Cleveland Heights 18, O. (F) ('37)

Rossman, Max, M.D.; Allentown State Hosp., Allentown, Pa. ('38)

*Rotн, William F., Jr., M.D.; Lt. Comdr., MC, USNR; c/o Franklin, Tenn. (F) ('35)

Ruggles, Arthur H., M.D.; Butler Hospital, Providence 6, R. I. (F) ('29)

*Russell, John A., M.D.; Lt., MC, USNR; c/o 4139 Norton Ave., Oakland 2, Calif. ("40)

Sanders, Helen; National Comm. for Mental Hygiene, I 790 Broadway, New York I9 ('42)

Schaefer, Phyllis D., M.D.; 6009 E. Seaside Walk, Long Beach 3, Calif. ('4I)

Schapiro, Joseph, M.D.; Bur. Child Guidance, 3514 Palisade Ave., Union City, N. J. ('35)

Schlezinger, Nathan S., M.D., Jefferson Medical College; 255 So. 17 th St., Phila., Pa. ('44)

Schneider, Wilmot F., M.D.; Babies \& Children's Hosp., Cleveland 6, O. ('40)

Schrag, A. Ralph, M.D.; Provincial Mental Inst., Edmonton, Alberta, Canada ('4I)

*Schroeder, Paul L., M.D.; Lt. Col., MC, Hq., 4th Service Command, Atlanta 3, Ga. (F) ('30)

Schumacher, Henry C., M.D.; Guidance Center, 2525 Euclid Ave., Cleveland 15, O. (F) ('26)

*Schwartz, Louis A., M.D.; Lt. Comdr., MC, USNR, U.S.S. Refuge, A.H. I , FPO, San Francisco, Calif, (F) ('31)

Scoville, Mildred C.; The Commonwealth Fund, 4 I E. 57th St., New York 22 (F) ('26)

Sears, Robert R., Ph.D., Child Welf. Res. Sta., Univ. of Iowa; I 124 Dill St., Iowa City, lowa ('45)

Seeberg, Elizabeth, Ph.D.; Norwood \& Valley Schools, I47 Mason St., Cincinnati I9, O. ('28)

SEl by, Nathaniel E., M.D., Neurological Inst.; 133 E. 58th St., New York 22 ('4I)

Seling, Lowell S., M.D., Recorders Court; 16196 Cherrylawn Ave., Detroit, Mich. (F) ('3o)

Senior, Rose D., M.D.; 5612 S. Kenwood Ave., Chicago 37, Ill. ('35)

Senn, Milton J. E., M.D.; Payne Whitney Clinic, N. Y. Hosp., 525 E. 68th St., New York 2 I ('45)

Sha kow, David; Worcester State Hospital, Worcester 4, Mass. (F) ('30)

*Shaskan, Donald, M.D.; Capt., MC, Bushnell Gen. Hosp., Brigham City, Utah ('43)

Sheldon, Sybil F.; 235 Marguerite Lane, Pasadena 2, Calif. ('42)

Shirley, Hale F., M.D.; Stanford Univ. Hosp., Clay \& Webster Sts., San Francisco 15, Calif. (F) ('4I)

ShIrLey, Mary M., Ph.D.; Psychological Clinic, Indiana University, Bloomington, Ind. ('42)

Siegel, Mrs. Miriam G.; Jewish Board of Guardians, 228 E. I gth St., New York 3 ('44)

Siewers, Albert B., M.D., Board of Ed.; 713 E. Genessee St., Syracuse 2, N. Y. (F) ('29)

Silveira, Aníbal, M.D.; Hospital de Juguerí, Estação de Juguerí S.P.R., São Paulo, Brazil ('4I)

Silverman, Baruch, M.D.; Mental Hygiene Inst., 53I Pine Ave. W., Montreal, Canada (F) ('3I)

*Silverman, Simon S.; Lt. AUS (0509330) AFIS; c/0 73-22 I92nd St., Flushing, N. Y. ('40)

Sims, Audrey; Guidance Center, 2525 Euclid Ave., Cleveland I 5, O. ('40)

Slavson, Samuel R.; Jewish Board of Guardians, 228 E. Igth St., New York 3 ('44)

Slawson, John, Ph.D., The American Jewish Committee; I9 E. 88th St., New York 28 (F) ('36)

Smalley, Ruth; Univ. of Pittsburgh, 2206 Cathedral of Learning, Pittsburgh 13, Pa. (F) ('36)

SMIth, Barry C.; The Commonwealth Fund, 4I E. 57th St., New York $22(\mathrm{H})$ ('30)

SмIтH, Groves Blake, M.D.; Beverly Farms, Inc., Godfrey, Ill. (F) ('30)

*Solomon, Joseph C., M.D.; Capt., MC, SCU I969, Ft. Missoula, Montana (F) ('38)

*Solomon, Philip, M.D.; Lt. Comdr., MC, USNR, 6th Med. Bn., 6th Mar. Div., c/o FPO, San Francisco, Calif. ("4I)

Sommer, Conrad, M.D.; Dept. Public Welf., Mental Hygiene Serv. 176 W. Adams St., Chicago 3, Ill. (F) ('38)

Spencer, Douglas, Ph.D., Office of Strategic Services, Washington, D. C.; c/o 400 E. 59th St., New York 22 ('32)

SPencer, Harvey, M.D.; Austen Riggs Foundation, Stockbridge, Mass. ('4I)

SPEYER, Helen; 70 E. 77 th St., New York 2I (F) ('37)

*Spiegel, Herbert X., M.D.; Capt., MC, Mason General Hosp., Brentwood, L. I., N. Y. ("45)

SpITz, Rene A., M.D.; i 50 Fifth Ave., New York 28 ('45)

*Stavsky, William H., Ph.D.; Capt., AGD, AUS; c/o 288 S. River St., Wilkes-Barre, Pa. ('42)

Steiner, Mrs. Lee R.; 114 W. 6ist St., New York 23 (F) ('38)

Steldern, Mrs. Florence G., Family Service Society; 293 S. Chester, Pasadena 5, Calif. ('43)

Stephenson, Charles W., M.D.; Box 62, Kennedy General Hosp., Memphis I5, Tenn. ('32) 
Syevenson, George S., M.D.; Nat'l Comm. for Men. Hyg., 1790 Broadway, New York I9 (F) (C) Stewart, Kathleen K., M.D., Langley-Porter Clinic; I 17 Eighth Ave., San Francisco 22, Calif. ('45)

Stone, Sybil A.; U. S. Dept. of Labor, Children's Bureau, Washington, D. C. ('45)

*Stor, Edward G., Ph.D.; Lt., USNR, Room 33, Federal Office Bldg., San Francisco, Calif. (F) ('31) Stranahan, G. Marion, M.D.; i Gramercy Park, New York 3 (F) ('3I)

Strauss, Bernard V., M.D., Kings County Hosp.; I Hanson Pl., Brooklyn 17, N.Y. ('43)

Strecker, Edward A., M.D.; Pennsylvania Hosp., i I I No. 49th St., Phila. 39, Pa. (F) ('24)

Struthers, J. N. P., M.D., West Michigan Children's Center, Myrtle \& Wood Sts., Muskegon, Mich. ('43)

Suluivan, Harry Stack, M.D., William Alanson White Foundation; 9003 Bradley Blvd., Bethesda 14, Md. (F) ('30)

Sumner, Mary C.; Children's Memorial Clinic, Ioor E. Clay St., Richmond I9, Va. (F) ('26)

Swackhamer, Gladys V.; 1409 6th Ave., Apt. 6, New York ig ('40)

Swanson, Florence L., M.D.; Psychopathic Hosp., 4200 E. 9th Ave., Denver 7, Colo. ('42)

Swirt, Sarah H., Community Service Soc.; I035 Fifth Ave., New York 28 (F) ('26)

Sylvester, Emmy, M.D.; Michael Reese Hosp., 29th St. \& Ellis Ave., Chicago I6, III. (F) ('41)

Symonds, Percival M., Ph.D.; Teachers College, Columbia Univ., New York 27 (F) ('37)

*Szurek, Stanislaus A., M.D.; Lt. MC, USNR, U. S. Naval Hosp., Farragut, Idaho (F) ('40)

Taft, J. Jessie, Ph.D.; Pennsylvania School of Soc. Work, 2410 Pine St., Phila. 3, Pa. (F) ('25)

Talbor, Mira, Bureau of Child Guidance; Io Mitchell Place, New York (F) ("40)

Tallman, Frank F., M.D.; Comm. Mental Diseases, State Office Bldg., Columbua I6, O. (F) ('38)

Taussig, Helen P.; 235 E. 22nd St., New York io (F) ('3I)

Teagarden, Florence M., Ph.D., Univ. of Pittsburgh; 726 Gypsy Lane, Pittsburgh 16, Pa. (F) ('37)

*Thompson, Lloyd J., M.D.; Col. MC; c/o 66 Wall St., New Haven I I, Conn. (F) ('29)

*Thurrotr, John C., M.D.; Capt., MC, Mason General Hosp., Brentwood, L. I., N. Y. (F) ('31)

Tiвout, Nelly H. C., M.D.; Reynier Vinkeleskade 54, Amsterdam, Z., Holland ('40)

Tiebout, Harry M., M.D.; 215 Milbank Ave., Greenwich, Conn. (F) ('28)

Trmme, Arthur R., M.D., Pasadena Child Guid. Clinic; I930 Wilshire Blvd., Los Angeles 5, Calif. ('44)

Tolman, Ruth S., Ph.D.; 4420 5oth St., N.W., Washington I6, D. C. ('39)

Topping, Ruth, Home for Girls; 5024 Indiana Ave., Chicago, Ill. ('4I)

Tow LE, Charlotte, University of Chicago; 574I Kenwood Ave., Chicago 37, Ill. (F) ('30)

Tracy, Anna Belle; Sch. Applied Soc. Sci., Western Res. Univ., 2 I 17 Adelbert Rd., Cleveland 6, 0. (F) ('3o)

TRUiTt, Ralph P., M.D., Ment. Hyg. Soc. of Maryland; IOI $_{4}$ St. Paul St., Baltimore 2, Md. (F) ('30)

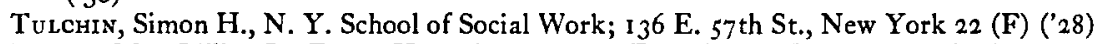

TurItz, Mrs. Lillian L., Foster Home Bureau; 310 E. 75 th St., New York 2I ('45)

*Uhler, Claude, M.D.; Major, MC, Letterman General Hosp., San Francisco, Calif. ('39)

UlRICH, Carl F., M.D., Western Res. Univ. Sch. of Med.; I6I 5 Hazel Dr., Cleveland 6, O. (F) ('30)

Valentine, Ruth, Ph.D.; USPHS Dispensary, 4th \& D St. N.W., Washington, D. C. (F) ('4I)

VANDer VeER, Adrian H., M.D.; Bobs Roberts Hosp., 920 E. 59th St., Chicago 37, Ill. (F) ('42)

Van Hyning, Mrs. Mary W.; I 270 South Washington St., Falls Church, Va. ('30)

Vedder, Mrs. Sarah, N. Y. Church Mission of Help; Rock Rimmon Rd., Stamford, Conn. ('3r)

Vosk, Mrs. Jeannette S., Bureau of Child Guidance; 167 E. 95th St., New York 28 ('42)

*Wadsworth, Morton L., M.D.; Capt., MC, AUS; c/o Dr. L. R. Hoelzle, Iogz Ivy Ave., St. Paul 6, Minn. ('4I)

Waelder, Robert, Ph.D., Bryn Mawr College; 2100 Walnut St., Phila., Pa. ('44)

Wallace, Dorothy; 6910 Reed Cottage Pl., Portland, Ore. ('4I)

Wannamaker, Claudia; Inst. for Juvenile Research, 907 S. Wolcott Ave., Chicago 12, Ill. (F) ('41)

Waterman, John H., M.D.; Bur. Mental Hygiene, 304 McIlhenny St., Houston 6, Texas ('42)

Watson, Goodwin, Ph.D.; Teachers College, Columbia Univ, 525 W. I 20 th St., New York 27 (F) ('33) 
"Weatherly, Howard E., M.D.; Capt. MC, i25th Sta. Hosp., APO 72, c/o Postmaster, San Fran. cisco, Calif. (' $4 \mathrm{I}$ )

WebB, Mrs. Evelyn R.; The Guidance Clinic, 129 E. Orange St., Lancaster, Pa. ('45)

WebBer, Alice J.; 221 W. 82 nd St., New York 24 (F) (3I)

Wechsler, David, Ph.D., Bellevue Hosp., Psychiatric Div.; 145 E. 92 nd St., New York 28 (F) (37)

WEILL, Blanche C., Ed.D.; 470 W. 24th St., New York II ('35)

WeIss, Theodore S., M.D., Bellevue Hosp., Psychiatric Div.; 27 W. 96th St., New York 25 ('4I)

Wells, Frederic L., Ph.D., Harvard University; 13 Holyoke St., Cambridge 38, Mass. (F) ('39)

Welsch, Exie E., M.D.; Guidance Center, 31 Gibbs St., Rochester 4, N. Y. (F) ('39)

Westburgh, Edward M., Ph.D.; The Institute, i I I North 49th St., Philadelphia 39, Pa. ('39)

Westlund, Norman, M.D., Saginaw Valley Chn's Center; I22 S. Charles St., Saginaw, Mich. ('4I)

Whitaker, Carl, M.D.; Oak Ridge Hospital, Oak Ridge, Tenn. ('42)

White, Blanche H.; Children's Home \& Service League, I825 W. Maple St., Wichita I2, Kan. ('40)

Wickman, E. K.; The Commonwealth Fund, 4 I E. 57th St., New York 22 (F) ('28)

Wickman, Katharine M.; Pediatric Psychiatric Clinic, Babies Hosp., I67th \& Broadway, New York 32 (F) ('29)

Wilderson, Myrna; Park Lane, Chagrin Falls, O. ('37)

Winters, Alfred G.; Children's Aid Society, IO427 Detroit Ave., Cleveland, O. ('42)

Winters, Ruth R.; Children's Aid Society, 10427 Detroit Ave., Cleveland, O. ('42)

Witmer, Helen L., Ph.D., Smith College Sch. for Soc. Work; 308 Quaker Lane, Alexandria, Va. (F) ('34)

Witmer, Lightner; Devon, Pa. ('25)

Witten berg, Rudolph M., Ph.D., Bureau of Child Guidance; 50 E. 89th St., New York 28 ("45)

Witten brook, John M., M.D.; Guidance Center, 2525 Euclid Ave., Cleveland I 5, O. ('44)

WolfF, Ernst, M.D.; 2535 Vallejo St., San Francisco 23, Calif. (F) ('39)

Wold.An, Kenneth I., S.Sc.D.; Connecticut Jr. Republic, Litchfield, Conn. ('40)

*Woltman, Adolf G.; Sgt. AUS; c/o I 5 E. I I6th St., New York 29 (F) ('39)

Wood, Louise; Dept. of Child Guidance, 704 Stanford Life Bldg., Jackson 27, Miss. (F) ('38)

Woods, Mrs. Lawrence C., Jr.; 270 Grant St., Swickley, Pa. (F) ('39)

Woodward, Luther E., Ph.D., Nat'l Comm. for Ment. Hyg.; 2400 Ave. M., Brooklyn io, N. Y. (F) ('40)

Woolr, Henriette, Bureau of Child Guidance; 237 W. I 5 th St., New York II ('42)

Woolley, Lawrence F., M.D.; 2030 Park Ave., Baltimore 17, Md. (F) ('32)

*Wortis, Joseph, M.D.; USPHS (R); 152 Hicks St., Brooklyn 2, N. Y. ("43)

Wyatt, Frederick, Ph.D.; I 7 Wright St., Cambridge 38, Mass. ('45)

Yarnell, Helen, M.D.; Mental Hygiene Clinic, I 1 I E. Front St., Plainfield, N. J. ('40)

Young, Paul C., Ph.D.; Dept. Psychology, Louisiana State University, Baton Rouge 3, La. ('37)

Younc, Robert A., Ed.D., Judge Baker Guidance Center; 17 Oak Knoll, Arlington 74, Mass. (F) ('40)

Zeithin, Goldie R., Bureau of Child Guidance; 130 W. 12th St., New York I I ('4I)

Zilboorg, Gregory, M.D., Psychoanalytic Inst.; 14 E. 75 th St., New York 21 (F) ('32) 